\title{
QUANTUM THEORY OF MAGNETIC ELECTRON LENSES BASED ON THE DIRAC EQUATION
}

\author{
R. JAGANNATHAN, R. SIMON, E.C.G. SUDARSHAN ${ }^{1}$ \\ Institute of Mathematical Sciences, C.I.T.Campus, Tharamani, Madras 600 113, India
}

and

N. MUKUNDA ${ }^{2}$

Centre for Theoretical Studies, Indian Institute of Science, Bangalore 560012, India

\begin{abstract}
A quantum theory of magnetic electron lenses based on a convenient formulation of the Dirac theory is outlined. It is shown that the passage from the conventional scalar theory to the spinor theory can be accomplished through a simple algebraic rule in analogy with the passage from scalar to vector light optics.
\end{abstract}

The conventional description of the quantum mechanics of electron optical image formation is based on a semiclassical treatment of the nonrelativistic Schrödinger equation [1,2], and is modeled after the traditional Fresnel-Kirchhoffintegral approach in light optics. In recognition of the fact that there are lenses where one is dealing with electrons at relativistic energies the description is rendered "relativistic" at the end by using relativistically corrected expressions for the mass and de Broglie wavelength of the electron [3].

It is only recently [4] that an attempt has been made to treat the positive energy electrons participating in the imaging process properly, on the basis of the Dirac equation. But even this treatment falls short of the goal for it leads ultimately to the suggestion that the spin can be regarded as a spectator degree of freedom and hence the space-time dependence can be handled through the Klein-Gordon propagator for the evolution of the wavefunction along the system (or optic) axis, obtained through a generalization of the semiclassical approach of Glaser's nonrelativistic theory.

In this context it is useful to recall the following aspect of the present status of high voltage electron microscopy [ 5 ]. Instruments used for the examination of thick specimens operate at accelerating voltages of the order of a few megavolts. While these are largely scaled up versions of the standard $100 \mathrm{kV}$ designs, they fail to achieve a resolving power comparable to that of the best $100 \mathrm{kV}$ microscopes. This is contrary to the expectation that the resolving power should increase with decreasing wavelength. Thus it becomes clear that even though a semiclassical treatment using the scalar wavefunction is successful in electron microscopy with low energy beams naive generalizations of such an approach fail to lead to optimal design at higher energies.

There exists an interesting analogy with light optics which may help to place the present paper in proper perspective. Though light is a vector wave one often uses with a fair amount of success a scalar theory in the practical design of optical systems. It may be argued, in partial recognition of the vector nature of light, that the light wave under consideration is polarized in the same direction everywhere and the scalar wavefunction

\footnotetext{
' Also at the Center for Particle Theory, University of Texas, Austin, TX, USA.

${ }^{2}$ Jawaharlal Nehru Fellow.
} 
used represents the amplitude of the wave thus polarized [6]. A moment's reflection shows that such an argument is untenable, for Maxwell's equations couple the space-time dependence of the field vectors to their polarization in a non-trivial way; the constraint part of the Maxwell equations, namely $\boldsymbol{\nabla} \cdot \boldsymbol{E}=\boldsymbol{\nabla} \cdot \boldsymbol{B}=0$, demands that the field vectors have no spatial variation in their respective directions of polarization and rules out from consideration even a simple spherical wave converging to (diverging from) a point. Thus, any attempt to analyse or affect the spatial variation of these vectors without due respect to their (space-time dependent) polarization will be inconsistent with these equations, notwithstanding the fact that in some practical cases this inconsistency may not affect the optimal design. A systematic procedure for handling spatial variation (modulation ) and polarization in a uniform way has been developed recently [7]. This procedure leads to a simple algebraic rule by which every time the spatial dependence is changed the polarization is readjusted in such a manner that consistency with the Maxwell equations is guaranteed. In the case of the "linearly" polarized gaussian beam, it predicts a component of the field vector along the beam axis and cross-polarization component in addition to the principal polarization component [8]. Such a cross-polarization component has indeed been observed experimentally [9].

The Dirac equation couples the amplitudes of the component of the four-spinor to their space-time variation. The conventional description of electron microscopy corresponds to a scalar theory wherein one component of the spinor is taken as the scalar wavefunction on whose spatial dependence the lens system acts, thus ignoring the spin degree of freedom and the subtle way in which the Dirac equation couples the spinor components. The analogy with electromagnetic waves suggests that we look for a convenient formulation of the Dirac theory leading to an algebraic rule which treats the spin and spatial degrees of freedom of the electron in a uniform way consistent with the Dirac equation. In this Letter we outline such a simple and straightforward approach to the quantum theory of electron optics in the case of magnetic lenses. As an illustration of the basic theory developed we shall demonstrate the focusing action of an axially symmetric thin magnetic lens on a narrow beam of practically monoenergetic electrons moving close to the system axis (paraxial) beam, using the lowest order approximation. Thus, for the first time, a quantum mechanical derivation of the classical formula of Busch [10] for the focal length of an axially symmetric thin magnetic lens is obtained, and the wellknown image rotation is accounted for, on the basis of the Dirac equation. Since the imaging part of an electron microscope invariably uses the magnetic lens system, we restrict our attention to the magnetic case. Generalization to lenses comprised of both magnetic and electric fields is straightforward.

A magnetic electron lens with rotational symmetry about its optical axis, say z-axis, is formed by a static magnetic field $B(x)$ for which the potential is usually chosen as

$\boldsymbol{A}(\boldsymbol{x})=(-A(\boldsymbol{x}) y, A(\boldsymbol{x}) x, 0)$,

$A(x)=\frac{1}{2} B(z)-\frac{1}{16} \frac{\mathrm{d}^{2} B(z)}{\mathrm{d} z^{2}} x_{\perp}^{2}+\frac{1}{384} \frac{\mathrm{d}^{4} B(z)}{\mathrm{d} z^{4}} x_{\perp}^{4}-\ldots$,

with the function $B(z)$ characterizing the field along the system axis such that

$\boldsymbol{B}(0,0, z)=B(z) \boldsymbol{k}$,

where $k$ is the unit vector along the z-axis. For a paraxial beam the effective field is confined to the paraxial region and correspondingly one can take in that case

$\boldsymbol{A}(\boldsymbol{x}) \approx\left(-\frac{1}{2} B(z) y, \frac{1}{2} B(z) x, 0\right)$.

In this prescription for the lens field the field due to the space-charge of the propagating electron beam itself has been ignored. In principle, there is no sharp boundary for the lens defined above. But, in practice, the lens can be thought of as situated in a finite interval on the z-axis, say between $z=z_{\ell}$ and $z=z_{\mathrm{r}}$; i.e. the field $B$ is concentrated within the region bounded by transverse planes at $z=z_{\ell}$ and $z=z_{\mathrm{r}}$, and the regions $\left(z<z_{\ell}\right)$ and $\left(z>z_{\mathrm{r}}\right)$ are practically field-free with $B(z)$ almost vanishing [11]. 
The central problem is to study the propagation of the electron beam along the optical axis of the system. The four-component spinor wavefunction of the beam is governed by the Dirac equation

$$
\begin{aligned}
& \mathrm{i} \hbar \frac{\partial \Psi(\boldsymbol{x}, t)}{\hat{a} t}=\left(m c^{2} \beta+c \alpha \cdot \hat{\boldsymbol{x}}\right) \Psi(\boldsymbol{x}, t), \\
& \hat{\boldsymbol{\pi}}=\hat{\boldsymbol{p}}+e A / c, \quad \beta=\left(\begin{array}{ll}
I & J \\
0 & J
\end{array}\right), \quad \alpha=\left(\begin{array}{ll}
0 & \sigma \\
0 & 0
\end{array}\right) .
\end{aligned}
$$

We are dealing with the scattering states of the system and are concerned only with almost paraxial quasimonoenergetic beams moving in the $+z$ direction. Hence, the beam wavefunction is of the form

$$
\begin{aligned}
\Psi(\boldsymbol{x}, t) & =\underset{p_{0}-\mathrm{Ap}}{\mathrm{j}} \mathrm{j} \quad \mathrm{d} p \exp [-\mathrm{i} E(p) t / \hbar] \psi(\boldsymbol{x} ; p), \ll p_{0}, \\
E(p) & =+\left(m^{2} c^{4}+c^{2} p^{2}\right)^{1 / 2},
\end{aligned}
$$

with

$$
\begin{aligned}
& \psi\left(\boldsymbol{x}_{\perp}, z<z_{\ell} ; p\right)=\int \mathrm{d}^{2} p_{\perp}\left[a_{+}(\boldsymbol{p}) u_{+}(\boldsymbol{p})+a_{-}(\boldsymbol{p}) u_{-}(\boldsymbol{p})\right] \exp (\mathrm{i} \boldsymbol{p} \cdot \boldsymbol{x} / h), \\
& \boldsymbol{p}=\left(\boldsymbol{p}_{\perp},+\left(p^{2}-p_{\perp}^{2}\right)^{1 / 2}\right),
\end{aligned}
$$

where $\left\{\boldsymbol{u}_{ \pm}(\boldsymbol{p}) \exp [\mathbf{i}(\boldsymbol{p} \cdot \boldsymbol{x}-E t) / \hbar\}\right.$ rre the standard positive energy four-component plane wave solutions of the free Dirac equation and $p_{0}$ is the mean momentum of the beam electrons. Further, since the beam electrons always move in the forward z-direction the potential should not contain Fourier components comparable to $p_{0} / \hbar$ so that no transitions between free-particle states with momentum differences of the order of $p_{0}$ are possible. Thus, the relative changes in $A_{\perp}$ over distances of the order of the de Broglie wavelength, $\hbar / p_{0}$, are small compared to unity. These conditions are expected to be met in practical lens systems, particularly in high voltage electron microscopes [4].

In an electron microscope, $\Psi\left(x_{\perp}, z<z_{\ell}, t\right)$ defined through (5), (6) represents the input wavefunction for the objective lens corresponding to the beam emerging from the aperture after being scattered by the specimen examined; and

$p_{0}=\left[2 e m V\left(1+e V / 2 m c^{2}\right)\right]^{1 / 2}=\left(2 e m V^{*}\right)^{1 / 2}$

the momentum of an electron accelerated through the operating voltage $V$ from rest. The spread in $p$-values around $p_{0}$, entering the integral in (5), is due to the inhomogeneities in the initial velocities of the electrons before acceleration and fluctuations in $V$, as well as to the partially inelastic scattering of the beam by the specimen examined.

Since the system is stationary with time-independent Dirac hamiltonian it is clear from (4) and (5) that the time-Fourier coefficient $\psi(\boldsymbol{x} ; p)$ of $\Psi(\boldsymbol{x}, t)$ has to satisfy the time-dependent equation

$$
\left[E(p)-m c^{2} \beta-c \alpha_{\perp} \cdot \hat{\boldsymbol{\pi}}_{\perp}+c \alpha_{z} \mathrm{i} \hbar \partial / \partial z\right] \psi\left(\boldsymbol{x}_{\perp}, z ; p \rightarrow 0 .\right.
$$

Integrating (7) for the $z$-evolution of $\psi$ as

$\psi\left(z^{\prime \prime} ; p\right)=\hat{G}\left(z^{\prime \prime}, z^{\prime} ; p\right) \psi\left(z^{\prime} ; p\right)$,

one gets the required z-evolution of $\Psi$ :

$\Psi\left(z^{\prime \prime}, t\right)=\int \mathrm{d} p \exp [-\mathrm{i} E(p) t / \hbar] \hat{G}\left(z^{\prime \prime}, z^{\prime} ; p\right) \psi\left(z^{\prime} ; p\right)$.

In the practically monoenergetic situation with mean momentum $p_{0}$ we have 
$\Psi\left(z^{\prime \prime}, t\right) \approx \hat{G}\left(z^{\prime \prime}, z^{\prime} ; p_{0}\right) \Psi\left(z^{\prime}, t\right)$.

In order to study the $z$-evolution of $\psi$ we shall rewrite (7) in a form very similar to the Dirac equation (4), with $\mathrm{z}$ taking the place of $t$ now; multiply throughout by $\alpha_{z}$, rearranging the terms, and defining

$\psi^{\prime}=M \psi, \quad M=\frac{1}{\sqrt{2}}\left(I+\chi \alpha_{z}\right), \quad \chi=\left(\begin{array}{cc}\xi & 0 \\ 0 & -1 / \xi\end{array}\right), \quad \xi(p)=\frac{E(p)+m c^{2}}{c p}$,

it can be seen that

$\mathrm{i} \hbar \frac{\partial \psi^{\prime}}{\partial z}=\hat{H}_{0} \psi^{\prime}, \quad H_{0}=(-p \beta+\overline{\mathcal{O}}), \quad \hat{\theta}=\chi \boldsymbol{\alpha}_{\perp} \cdot \hat{\boldsymbol{\pi}}_{\perp}$.

Now, it may be noted that

$\hat{H}_{0}^{2}=p^{2}+\mathcal{O}^{2}=p^{2}-\hat{\pi}_{\perp}^{2}-\frac{2 e}{c} B_{z} S_{z}, \quad S=\left(\begin{array}{ll}\sigma & 0 \\ 0 & \sigma\end{array}\right)$,

where $B_{z}$ is the $z$-component of the lens field given by

$B_{z}(x)=B(z)-\frac{1}{4} \frac{\mathrm{d}^{2} B(z)}{\mathrm{d} z^{2}} x_{\perp}+\frac{1}{64} \frac{\mathrm{d}^{4} B(z)}{\mathrm{dz}^{4}} x_{\perp}-\ldots \approx B(z) \quad$ (forparaxial beam).

In geometrical electron optics the z-evolution of phase-space coordinates $\left(\boldsymbol{x}_{\perp}, \mathrm{d} \boldsymbol{x}_{\perp} / \mathrm{d} z\right)$ is governed by the hamiltonian $-\left(p^{2}-\pi_{\perp}^{2}\right)^{1 / 2}$ and it is the power series expansion in terms of $\pi_{\perp}^{2} / p^{2}$ that helps to study the system under successive approximations, the paraxial approximation followed by aberrations of various orders [12]. Looking at (12) and (13) suggests we look for a power series expansion of (12) in terms of $\hat{\boldsymbol{x}}_{\perp} /$ that could lead naturally to the geometrical optics description in the classical limit. To obtain the desired power series expansion of (12) we proceed as follows. It is seen easily that for any solution $\psi^{\prime}$ of (12) representing a paraxial beam propagating in the $+\mathrm{z}$ direction the upper pair of components is large compared to the lower pair of components irrespective of the value of p. Thus, in (12) 79 is an "odd" operator that couples the large and small components of $\psi^{\prime}$ and $f t$ is "even" which does not not make such a coupling of the large and small components. The situation is similar to the case of the Dirac equation (4) for which any nonrelativistic positive energy solution has the upper pair of components large compared to the lower pair of components. Recalling that to analyse the Dirac equation (4) as nonrelativistic part + relativistic corrections the systematic procedure is the Foldy-Wouthuysen technique [13] of eliminating the odd operators, it is realised that to analyse (12) as paraxial part + nonparaxial corrections we should adopt a similar technique that leads to the elimination of the odd operators from (12).

In view of the above observation, let us define

$\psi_{0}=\hat{T} \psi^{\prime}, \quad \hat{T}=\exp \left[-\frac{1}{2} \tanh ^{-1}(\beta \hat{\mathcal{O}} / p)\right]=\exp \left(-\beta \hat{\mathcal{O}} / 2 p+\beta \hat{\mathcal{O}}^{3} / 6 p^{3}-\ldots\right)$.

Then, with

$$
\begin{aligned}
\mathscr{H}_{0} & =-\left(p^{2}+\hat{\mathscr{C}}^{2}\right)^{1 / 2}=-\left\{p^{2}-\left[\hat{\pi}_{\perp}^{2}+(2 e / c) B_{z} S_{z}\right]\right\}^{1 / 2} \\
& =-p+\left(1 / 2 p\left[\hat{\pi}_{\perp}^{2}+(2 e / c) B_{z} S_{z}\right]+\left(1 / 8 p^{3}\right)\left[\hat{\pi}_{\perp}^{2}+(2 e / c) B_{z} S_{z}\right]^{2}+\ldots,\right.
\end{aligned}
$$

eq. (12) becomes

$\mathrm{i} \hbar \frac{\partial \psi_{0}}{\partial z} \approx\left(\hat{T} \hat{H}_{0} \hat{T}^{-1}\right) \psi_{0}=\beta \hat{\mathscr{H}}_{0} \psi_{0}$,

neglecting terms comparitively small in view of the z-dependence of $\psi$ being largely due to exp (ipz/) $i$ and the relative changes in $\boldsymbol{A}_{\perp}$ over distances of the order of $h / p$ being small compared to unity. 
It is to be noted that the z-evolution of the Dirac $\psi$ governed by (7), or equivalently (11), (12), is not unitary. On the other hand, $\psi_{0}$ has almost unitary z-evolution as seen from $(16),(17)$, or in other words, $\int \mathrm{d}^{2} x_{\perp} \psi_{0}^{\dagger} \psi_{0}$ is almost conserved along the z-axis.

We are interested in obtaining the expression for $\hat{G}\left(z^{\prime \prime}>z_{\mathrm{r}}, z^{\prime}<z_{\ell}\right)$ that relates the Dirac $\psi$ 's in the input (object) space $\left(z<z_{\ell}\right)$ and the output (image) space $\left(z>z_{\mathrm{r}}\right)$. In the absence of the lens field eq. (17) is exact and correspondingly for any free space solution $\psi_{0}$ representing a beam moving in the forward z-direction the lower pair of components become zero. Hence, for the input wavefunction in our problem we can assume the condition

$\beta \psi_{0(\text { in })}\left(z<z_{\ell}\right)=\psi_{0(\text { in })}\left(z<z_{\ell}\right)$,

as can be verified directly from (6), (11) and (15). Thus, to obtain $\hat{G}\left(z^{\prime \prime}<z_{\mathrm{r}}, z^{\prime}<z_{\ell}\right)$ one can integrate (17) formally, dropping $f t$ which commutes with $\hat{\mathscr{H}}_{0}$, and go back to the Dirac $\psi$ representation. The results is as follows: Let

$\hat{h}_{z}=+\left(p^{2}-\hat{p}_{\perp}^{2}\right)^{1 / 2}=p-(1 / 2 p) \hat{p}_{\perp}^{2}-\left(1 / 8 p^{3} \hat{p}_{\perp}^{4}-\ldots\right.$.

With

$\hat{\mathscr{T}}=\hat{T}($ free $) M=\left\{\exp \left[-\frac{1}{2} \tanh ^{-1}\left(\beta \chi \alpha_{\perp} \cdot \boldsymbol{p}_{\perp} / p\right)\right]\right\} M$,

define

$$
\begin{aligned}
\hat{\boldsymbol{Q}}_{\perp} & =\hat{\mathscr{T}}^{-1} \boldsymbol{x}_{\perp} \hat{\mathscr{T}}=\boldsymbol{x}_{\perp}-\frac{1}{2} \mathrm{i} h \hat{h}_{z}^{-1}\left\{\beta \chi \boldsymbol{\alpha}_{\perp}+\hat{h}_{z}^{-1}\left(\hat{h}_{z}+p\right)^{-1}\left[\beta \chi\left(\boldsymbol{\alpha}_{\perp} \cdot \hat{\boldsymbol{p}}_{\perp}\right) \hat{p}_{\perp}+(2 \mathrm{i} / \hbar) \hat{h}_{z} S_{z}\left(\boldsymbol{k} \times \hat{\boldsymbol{p}}_{\perp}\right)\right]\right\} \\
& =\boldsymbol{x}_{\perp}-\mathrm{i}(\hbar / 2 p) \beta \chi \boldsymbol{\alpha}_{\perp}+\ldots,
\end{aligned}
$$

and

$\mathscr{S}_{z}=\hat{\mathscr{T}}^{-1} S_{z} \hat{\mathscr{T}}=\hat{h}_{z}^{-1}\left(p S_{z}-\beta \chi \alpha_{z} \boldsymbol{S} \cdot \hat{\boldsymbol{p}}_{\perp}\right)=S_{z}-(1 / p) \beta \chi \alpha_{z} S \cdot \hat{\boldsymbol{p}}_{\perp}+\ldots$,

and note that $\hat{\mathscr{T}}^{-1} \boldsymbol{p}_{\perp} \hat{\mathscr{T}}=\hat{\boldsymbol{p}}_{\perp} \cdot$ Then we can write

$$
\begin{aligned}
& \psi_{\text {(out) }}\left(z^{\prime \prime}>z_{\mathrm{r}}\right)=\hat{G}\left(z^{\prime \prime}>z_{\mathrm{r}}, z^{\prime}<z_{\ell}\right) \psi_{(\text {in })}\left(z^{\prime}<z_{\ell}\right), \\
& \hat{\mathscr{G}}\left(z^{\prime \prime}>z_{\mathrm{r}}, z^{\prime}<z_{\ell}\right)=\hat{G}_{\mathrm{F}}\left(z^{\prime \prime}, z_{\mathrm{r}}\right) \hat{G}\left(z_{\mathrm{r}}, z_{\ell}\right) \hat{G}_{\mathrm{F}}\left(z_{\ell}, z^{\prime}\right) \\
& \quad=\exp \left[-\left(\mathrm{i} / \hbar \mathrm{h}^{\prime \prime}-z_{\mathrm{r}}\right) \hat{\mathscr{H}}(\text { free })\right] \mathbb{P}\left\{\operatorname { e x p } ( \frac { \mathrm { i } } { \hbar } _ { z _ { \mathrm { r } } } ^ { \mathrm { J } } \mathrm { dz } \mathscr { H } ( \mathrm { z } ) \mathrm { j } \} \operatorname { e x p } \left[-\left(\mathrm{i} / \hbar\left(z_{\ell}-z^{\prime}\right) \hat{\mathscr{H}}(\text { free })\right],\right.\right.
\end{aligned}
$$

where

$$
\begin{aligned}
\hat{\mathscr{H}} & =\hat{\mathscr{T}}^{-1} \hat{\mathscr{H}}_{0} \hat{\mathscr{T}}=\hat{\mathscr{H}}_{0}\left(\boldsymbol{x}_{\perp} \rightarrow \hat{\boldsymbol{Q}}_{\perp}, S_{z} \rightarrow \hat{\mathscr{S}}_{z}\right) \\
& =-p+(1 / 2 p)\left\{\left[\hat{\boldsymbol{p}}_{\perp}+(e / c) \boldsymbol{A}_{\perp}\left(\hat{\boldsymbol{Q}}_{\perp}, z\right)\right]^{2}+(2 e / c) B_{z}\left(\hat{\boldsymbol{Q}}_{\perp}, z\right) \hat{\mathscr{S}}_{z}\right\} \\
& +\left(1 / 8 p^{3}\right)\left\{\left[\hat{\boldsymbol{p}}_{\perp}+(e / c) \boldsymbol{A}_{\perp}\left(\hat{\boldsymbol{Q}}_{\perp}, z\right)\right]^{2}+(2 e / c) B_{z}\left(\hat{\boldsymbol{Q}}_{\perp}, z\right) \hat{\mathscr{S}}_{z}\right\}^{7} \ldots,
\end{aligned}
$$

the subscripts $\mathrm{F}$ and $\mathrm{L}$ denote respectively the "free propagation" and "lens action", and the symbol $\mathrm{P}$ stands for z-ordering.

The relation between $\phi$ 's at any two arbitrary transverse planes along the optic axis, with one or both of them immersed in the lens, can also be obtained by integrating (17) formally and reverting to the Dirac representation. But the result cannot be expressed in a form as simple as in (23), (24). Further, let us note the following: Since $\left(\hat{\boldsymbol{x}}_{\perp}, \boldsymbol{p}_{\perp}\right) \rightarrow\left(\hat{\boldsymbol{Q}}_{\perp}, \hat{\boldsymbol{p}}_{\perp}\right)$ is a similarity transformation, $\hat{\boldsymbol{Q}}_{\perp}$ and $\boldsymbol{p}_{\perp}$ are canonically conjugate and the new transverse position operator $\hat{\boldsymbol{Q}}_{\perp}$ corresponds to a "mean" (or classical) observable like in the FoldyWouthuysen theory [13]. The nonhermitian nature of $\hat{\boldsymbol{Q}}_{\perp}$ reflects the nonunitary z-evolution of the Dirac $\psi$. Similar remarks hold for $S_{z} \rightarrow \mathscr{S}_{z}$. 
As an application of the above formalism we shall now understand the focusing of a practically monoenergetic paraxial beam of electrons by an axially symmetric thin magnetic lens. To do this we shall consider the paraxial approximation of (23), (24). In this case the effective lens field is paraxial corresponding to the potential given by (3) and so $B_{z} \approx B(z)$. Further, in view of the paraxial nature nature of the beam, we shall retain in (24) only the first two terms, considering as negligible the terms of order $\left(1 / p^{3}\right)$ and higher powers of the expansion parameter $(1 / p)$. Consistently, in subsequent calculations we have to consider the terms of order $\left(1 / p^{3}\right)$ and higher powers of $(1 / p)$ as small compared to terms of order up to $\left(1 / p^{2}\right)$. Thus, under the paraxial approximation we get

$$
\begin{aligned}
& \mathscr{H} \approx \mathscr{H}^{1}=-p+(1 / 2 p) \hat{p}_{\perp}^{2}+\left(e^{2} B^{2} / 8 p c^{2}\right) x_{\perp}^{2}-\left(\mathrm{i} \hbar e^{2} B^{2} / 8 p^{2} c^{2}\right) \beta \chi \alpha_{\perp} \cdot \boldsymbol{x}_{\perp} \\
& \quad+(e B / 2 p c) L_{z}+(e B / p c) S_{z^{-}}\left(e B / 2 p^{2} c\right) \beta \chi \alpha_{z} \boldsymbol{S}_{\perp} \cdot \boldsymbol{p}_{\perp}, \\
& \hat{H}(\text { free }) \approx \hat{H}^{1}(\text { free })=-p+(1 / 2 p) \hat{p}_{\perp}^{2} .
\end{aligned}
$$

It can be verified that $\hat{\mathscr{H}}^{1}$ commutes only with $J_{z}=\left(L_{z}+S_{z}\right)$, not separately with $L_{z}$ and $S_{z}$, as it should be in the Dirac theory for a system with rotational symmetry about the z-axis.

Using the paraxial approximation (25) for the effective hamiltonian $\mathscr{H}$ in $(24)$, and approximating the lens propagator $\hat{G}_{\mathrm{L}}$ by its first order expression, we can write following (10)

$$
\begin{aligned}
& \Psi_{\text {(out) }}\left(z^{\prime \prime}\right) \approx\left[\hat{G}_{\mathrm{F}}^{1}\left(z^{\prime \prime}, z_{\mathrm{r}} ; p_{0}\right)\right]\left[\hat{G}_{\mathrm{L}}^{1}\left(z_{\mathrm{r}}, z_{\ell} ; p_{0}\right)\right]\left[\hat{G}_{\mathrm{F}}^{1}\left(z_{\ell}, z^{\prime} ; p_{0}\right)\right] \Psi_{(\text {in) }}\left(z^{\prime}\right) \\
& \quad=\left[\exp \left\{(\mathrm{i} / \hbar)\left(z^{\prime \prime}-z_{\mathrm{r}}\right)\left(p_{0}-\hat{p}_{\perp}^{2} / 2 p_{0}\right)\right\}\right] \\
& \quad \times\left[\exp \left\{(\mathrm{i} / \hbar)\left(z_{\mathrm{r}}-z_{\ell}\right)\left(p_{0}-\hat{p}_{\perp}^{2} / 2 p_{0}\right)-\left(\mathrm{i} p_{0} / \hbar\right)\left(x_{\perp}^{2} / 2 f\right)-\beta \chi\left(p_{0}\right) \boldsymbol{\alpha}_{\perp} \cdot \boldsymbol{x}_{\perp} / 2 f\right\}\right. \\
& \left.\quad \times \exp \left\{-(\mathrm{i} \theta / \hbar)\left(\hat{L}_{z}+S_{z}\right)\right\} \exp \left\{-(\mathrm{i} \theta / \hbar)\left(S_{z}-\beta \chi\left(p_{0}\right) \alpha_{z} S_{\perp} \cdot \hat{\boldsymbol{p}}_{\perp} / p_{0}\right)\right\}\right] \\
& \quad \times\left[\exp \left\{(\mathrm{i} / \hbar)\left(z_{\ell}-z^{\prime}\right)\left(p_{0}-\hat{p}_{\perp}^{2} / 2 p_{0}\right)\right\}\right] \Psi_{(\mathrm{in})}\left(z^{\prime}\right),
\end{aligned}
$$

for a practically monoenergetic beam of mean momentum $p_{0}$, where

$\frac{1}{f}=\frac{e^{2}}{4 p_{0}^{2} c^{2}} \int_{z \ell}^{z \mathrm{r}} \mathrm{d} z B^{2} \approx \frac{e}{8 m e^{2} V^{*}} \int_{-\infty}^{\infty} \mathrm{d} z B^{2}$,

and

$\theta=\frac{e}{2 p_{0} c} \int_{z_{\ell}}^{z_{\mathrm{r}}} \mathrm{d} z B=\left(\frac{e}{8 m c^{2} V^{*}}\right)^{1 / 2} \int_{z \ell}^{z_{\mathrm{R}}} \mathrm{d} z B$.

Let us now compute the Dirac current density at the output plane at $z=z_{\mathrm{r}}$ corresponding to the input wavefunction

$$
\begin{gathered}
\Psi_{(\text {in })}\left(x_{\perp}, z^{\prime}\right)=\left(c p_{0} \xi / 2 E\right)^{1 / 2}\left(\begin{array}{c}
a_{+} \\
a_{-} \\
a_{+} / \xi \\
a_{-} / \xi
\end{array}\right) \exp \left[\mathrm{i}\left(p_{0} z^{\prime}-E t\right) / \hbar\right], \quad z^{\prime}<z_{\ell}, \\
\left|a_{+}\right|^{2}+\left|a_{-}\right|^{2}=1,
\end{gathered}
$$

a plane wave of momentum $p_{0}$. The associated input current density is

$\boldsymbol{j}_{\text {(in) }}\left(x_{\perp}, z^{\prime}\right)=c \Psi_{(\text {in })}^{+} \alpha \Psi_{(\text {in })}=v(0,0,1) ; \quad z^{\prime}<z_{\ell}$,

where $v=c^{2} p_{0} / E$ is the velocity of the electrons. This current corresponds clearly to a system of rays parallel to the z-axis. The output wavefunction at the plane $z=z_{\mathrm{r}}$ is 
$\Psi_{\text {(out) }}\left(z_{\mathrm{r}}\right)=\hat{G}_{\mathrm{L}}^{\mathrm{L}}\left(z_{\mathrm{r}}, z_{\ell}\right) \Psi_{(\text {in })}\left(z_{\ell}\right)$,

as seem from (26) and (29). To compute $\boldsymbol{j}_{\text {(out) }}$ we shall assume that $z_{\mathrm{r}}-z_{\ell} \ll f$. This enables us to separate the factors containing $\boldsymbol{x}_{\perp}$ and $\hat{\boldsymbol{p}}_{\perp}$ in $\hat{G}_{\mathrm{L}}^{1}$ without regard to their noncommutativity. Then, up to first order in $\boldsymbol{x}_{1}$,

$\boldsymbol{j}_{\text {(out) }}\left(\boldsymbol{x}_{\perp}, z_{\mathrm{r}}\right)=c \Psi_{\text {(out) }}^{+} \alpha \Psi_{\text {(out) }} \approx v(-x / f,-y / f, 1)$,

with $f$ given by (27). Noting that $f$ is always positive, we see that the current density vector at every point in the transverse plane at $z_{\mathrm{r}}$ is pointing towards the point $\left(0,0, z_{\mathrm{r}}+f\right)$ on the $\mathrm{z}$-axis, the focal point. This corresponds to a system of radially converging rays and our system indeed acts as a converging lens of focal length $f$ for the Dirac current. The assumption $z_{\mathrm{r}}-z_{\ell} \ll f$ used above in the computation of $\boldsymbol{j}_{\text {(out) }}$ is seen to be precisely the "thin" lens approximation. Thus, we have derived the celebrated classical formula of Busch [10] for the focal length of an axially symmetric thin magnetic lens, as the lowest order approximation result in a formalism based on the Dirac equation. Further, the presence of the term $\exp \left(-\mathrm{i} \theta \hat{L_{z}} / \hbar\right)$ in $(26)$ explains the quantum mechanics of the well-known image rotation through the angle $\theta$ given by (28).

Eqs. (25)-(28) describe the paraxial optics (gaussian optics, or first-order optics) of the Dirac electrons in an axially symmetric magnetic lens and can, in fact, be called the generalized Huygens principle for this case, borrowing the terminology of light optics. Higher order corrections to these equations, resulting from a systematic inclusion of more terms in (21)-(24), will account for the aberrations suffered by the Dirac electron beam propagation when there are deviations from the ideal paraxial situation. It should be worthwhile to study the practical design aspects including the aberration calculations based on the above formalism and we shall return to the topic elsewhere.

To conclude let us make some observations:

As in the Foldy-Wouthuysen representation of the Dirac theory [13] it is $\psi_{0}$ that goes in the nonrelativistic limit into the Schrödinger-Pauli wavefunction, as can be seen by comparing (16), (17), with the nonrelativistic paraxial wave equation of Glaser [ 1], and with the spin terms disregarded in (16), (17) one gets the non-relativistic scalar theory. Now, if the $\psi_{0}$-equation (17) (without the spin terms) is taken to represent a scalar theory in the relativistic domain of $p$, the use of Klein-Gordon current density, which is given by the same formula as for the nonrelativistic Schrödinger current density, seems to lead to fairly satisfactory results in the lowest order, as for example in understanding the focusing by the thin lens, if the relativistic mass is used instead of the rest mass along with the use of the relativistic expression for the momentum or the de Broglie wavelength. This explains the apparent success of the nonrelativistic formulae in the relativistic domain also, when used with suitable substitution rules. But the proper quantum theory of relativistic electron lenses based on the Dirac equation should have the form as we have described above.

It is seen that the effective hamiltonian $\mathscr{H}$ in $(24)$ can be obtained from the classical hamiltonian $-\left(p^{2}-\pi_{\perp}^{2}\right)^{1 / 2}$ by (i) replacing $\pi_{\perp}^{2} \rightarrow\left(\alpha_{\perp} \cdot \pi_{\perp}\right)^{2}$ followed by (ii) a similarity transformation leading to the replacements $\boldsymbol{x}_{\perp} \rightarrow \hat{\boldsymbol{Q}}_{\perp}, S_{z} \rightarrow \hat{\mathscr{S}}_{\bar{z}}$.This is exactly analogous to the two step process found in the light optics where one passes from geometrical to wave optics through a "quantization" or "wavization" [ 14,15] and then from scalar to the vector Maxwell optics [7,8] through the replacement of $\boldsymbol{x}_{\perp}$ by a matrix operator.

Problems of classical theory of charged particle optics, related to imaging process and the ones associated with accelerator systems, have benefited substantially from Lie group and algebraic techniques [16]. These algebraic techniques have also been used to clarify several issues in light optics related to ray optics [17], scalar wave optics [18], and Maxwell optics [7,8]. Expression of the propagator $\vec{G}$ in operator form as in (23), (24) and (26) shows that such algebraic techniques retain their relevance in the quantum theory of electron optics as well.

Finally, it should be emphasized that, though for illustration only lenses with rotational symmetry about the $\mathrm{z}$-axis have been considered above the general formalism contained in (4)-(24) can also deal with any other type of magnetic lens with a straight optic axis, such as the quadrupole lens. 


\section{References}

[ 1 ] W. Glaser, in: Handbuch der Physik, Vol. 33, ed. S. Flügge (Springer, Berlin, 1956) p. 123.

[2 ] P. W. Hawkes, in: Computer processing of electron microscope images, ed. P. W. Hawkes (Springer, Berlin, 1980).

[3] R.D. Heidenreich, Fundamentals of transmission electron microscopy (Interscience, New York, 1964).

[4] H.A. Ferwerda, B.J. Hoenders and C.H. Slump, Opt. Acta 33 (1986) 145, 159.

[5] T. Mulvey, in: Magnetic electron lenses, ed. P.W. Hawkes (Springer, Berlin, 1982) p. 360; B.K. Vainshtein, Sov. Phys. Usp. 30 (1987) 393.

[6] M. Lax, W.H. Louisell and W.B. McKnight, Phys. Rev. A 11 (1975) 1365.

[7] N. Mukunda, R. Simon and E.C.G. Sudarshan, Phys. Rev. A 28 (1983) 2933; J. Opt. Soc. Am. A 2 (1985) 416 ; N. Mukunda, E.C.G. Sudarshan and R. Simon, J. Opt. Soc. Am. A 2 (1985) 1291.

[ 8 ] R. Simon, E.C.G. Sudarshan and N. Mukunda, J. Opt. Soc. Am. A 3 (1986) 356; Appl. Opt. 26 (1987) 1589.

[9] Y. Fainman and J. Shamir, Appl. Opt. 23 (1984) 3188.

[10] H. Busch, Ann. Phys. (Leipzig) 81 (1926) 974.

[11] P.W. Hawkes, ed., Magnetic electron lenses (Springer, Berlin, 1982).

[ 12] P.A. Sturrock, Static and dynamic electron optics (Cambridge Univ. Press, Cambridge, 1955).

[13] L.L. Foldy and S.A. Wouthuysen, Phys. Rev. 78 (1950) 29;

S. Tani, Prog. Theor. Phys. 6 (1951) 267;

R. Acharya and E.C.G. Sudarshan, J. Math. Phys. 1 (1960) 532.

[14] D. Marcuse, Light transmission optics (Van Nostrand, New York, 1972).

[ 15] V.I. Man'ko and K.B. Wolf, in: Lie methods in optics, eds. J. Sanchez Mondrag6n and K.B. Wolf (Springer, Berlin, 1986); T. Pradhan, Phys. Lett. A 122 (1987) 397.

[ 16] A.J. Dragt, E. Forest and K.B. Wolf, in: Lie methods in optics, eds. J. Sanchez Mondrag6n and K.B. Wolf (Springer, Berlin, 1986); K.B. Wolf, Ann. Phys. 172(1986) 1;

A.J. Dragt and E. Forest, in: Advances in electronics and electron physics, Vol. 67, ed. P.W. Hawkes (Academic Press, New York, 1986);

A.J. Dragt, Lectures in nonlinear orbit dynamics, American Institute of Physics Conference Proceedings, Vol. 87 (1982);

E.D. Courant and H.S. Snyder, Ann. Phys. 3 (1958) 1;

E.R. Caianiello, Nuovo Cimento 10 (1953) 581;

E.R. Caianiello and A. Turrin, CERN Report (CERN/T/ERC/3) 1953.

[17] M. Nazarthy and J. Shamir, J. Opt. Soc. Am. 72 (1982) 356;

A.J. Dragt, J. Opt. Soc. Am. 72 (1982) 372;

E.C.G. Sudarshan, R. Simon and N. Mukunda, Opt. Acta 32 (1985) 855

[ 18] H. Bacry and M. Cadilhac, Phys. Rev. A 23 (1981) 2533;

E.C.G. Sudarshan, R. Simon and N. Mukunda, Phys. Rev. A 28 (1983) 2921;

H. Bacry, in: Proc. XIII Int. Colloq. on Group theoretical methods in physics, ed. W.W. Zachary (World Scientific, Singapore, 1984). 